\title{
7. ZUSAMMENFASSENDE BEMERKUNGEN
}

Ohne Zweifel wird das hier vorliegende Corpus nicht nur den Forschungskomplex „Maximilianlehrbücher" verändern, sondern auch die internationale Glossarforschung um ein soziologisch bemerkenswertes mitteleuropäisches Kapitel erweitern. Es zeigt exemplarisch das systematisierte und aktive Interesse an Vulgärsprachen in höchsten Kreisen, dazu bestätigt es - mit einem Ausblick auf gedruckte Überlieferungen - ganz allgemein eine ...allmähliche Aufschwungphase von 1400 bis etwa $15 \% 0 \ldots{ }^{220}$. Allerdings gehen weder Holubařs Vokabular für Ladislaus Postumus noch die Trialogustradition zwischen 1489 und 1513 über eine nomenklatorische oder alphabetische Auflistung von Wörtern hinaus, wie einige teils ältere Überlieferungen, die auch zusammenhängende Texte in volgare bringen. Dieser italienischdeutsche Glossartyp, welcher neben Wortlisten auch Dialoge und Wendungen des Alltagslebens enthält, dürfte in Venedig seine Heimat gehabt haben. Ihm entspricht strukturell etwa der Vocabolario Quadrilingue im schon öfters genannten Mosbachischen Cod. Pal. lat $1789^{221}$. Die Höhepunkte sind das Werk Georgs von Nürnberg von 1424 und - mit einmaliger Strahlkraft ins sechzehnte Jahrhundert - die vorhin genannte Tradition nach Adam von Rottweil. Im Vergleich damit können die hier edierten Überlieferungen nicht als Sprachführer im modernen Sinn bezeichnet werden ${ }^{222}$.

Der Konnex unserer Glossare mit einem der hervorragendsten europäischen Herrscherhäuser schafft neben linguistischen zusätzliche kulturhistorische Perspektiven, von denen im Detail ja bereits gesprochen wurde. Herausgehoben und ergänzt seien noch vier Punkte:

1. Die „herrschaftlichen" Vokabulare sind erstaunlich pragmatisch auf den Fremdsprachenerwerb des Alltags zugeschnitten. Zwar steht das Lateinische in traditioneller Weise an erster Stelle, ist aber nicht mehr eindeutig als Zielsprache zu fixieren. Natürlich muß nach wie vor vom Lateinunterricht ausgegangen werden. Doch ist - nicht nur im

220 H. GLÜCK, Die Anfänge des DaF-Unterrichts. Deutsch als Fremdsprache im Mittelalter und in der frühen Neuzeit. Mitteilungen des deutschen Germanistikverbandes 47 (2000) 170. Eine Zusammenfassung bisheriger Forschungen bei A. Rossebastiano, La tradition des manuels polyglottes dans l'enseignement des langues, in: History of the Language Sciences = Geschichte der Sprachwissenschaften 1/1. Berlin-New York 2000, 688-698. Die neueste Übersicht über die tschechische Lexikographie am Beginn der Neuzeit bei KęDELska, Slowniki (zit. A. 2) 14-34 und passim.

${ }^{221}$ J. Kresálková, Il Vocabolario Quadrilingue Latino-Veneto-Ceco-Tedesco (Cod.Pal.Lat. 1789). Bergamo 1984,VII-VIII. Das erste gedruckte Buch dieser Art mit deutschem Leittext ist das Vocabularium Latinis, Gallicis et Theutonicis verbis scriptum aus Lyon von 1514. Ein überraschender Höhepunkt dieser Entwicklung ist dann das niederdeutsch-russische Lehrbuch von Tönnies Fenne, vgl. Tönnies Fenne's Low German Manual of Spoken Russian, Pskov 1607. Ed. L.L. Hammerich and R. Jakobson. II. Copenhagen 1970.

222 Vgl. O. Pausch, Das älteste italienisch-deutsche Sprachbuch (DS Österr.Akad. Wiss., phil.-hist. Kl. 111 = Veröffentlichungen der Historischen Kommission 1).Wien 1972, 38. Dazu zuletzt A. Rossebastiano, Deutsch-italienische Vokabulare des 15. Jahrhunderts, in: Die Volkssprachen als Lerngegenstand im Mittelalter und in der frühen Neuzeit. Akten des Bamberger Symposiums am 18. und 19. Mai 2001. Hrsg. von H. GLÜcK (Die Geschichte des Deutschen als Fremdsprache 3) Berlin - New York 2002, 4-19. 
Holubařtext - sicher das drittgereihte Tschechische am wichtigsten. Ladislaus Postumus sollte nach seiner Freigabe durch Kaiser Friedrich III. so schnell wie möglich diese Sprache lernen, und auch die Ambitionen Maximilians I. lagen - wie bereits erörtert - auf der Kompetenz von ... windisch und behamisch... . Die Lehrbücher verzichten völlig auf jede herkömmliche scholastisch-glossatorische Gelehrsamkeit und zeigen die Loslösung von schulmäßig geprägten Exegesen und Definitionen zur parole ${ }^{223}$. Diese Texte - wie möglicherweise auch das Glossar Johanns von Mosbach, der immerhin ein wittelsbachischer filius Ottonis ducis war, illustrieren offensichtlich das Durchschlagen der eingangs genannten Ideen der Goldenen Bulle bzw. des Aeneas Silvius Piccolomini. In diesem Zusammenhang scheint übrigens der im Spätmittelalter quantitativ führende Typ des Vocabularius ex quo keine Rolle gespielt zu haben.

Ein Blick auf einschlägige Bibliotheksbestände des ausgehenden Mittelalters oder auf das erste gedruckte lateinisch-tschechische Lexikon, den Lactifer des Johann Aquensis von 1511 läßt die Modernität dieses zweckbezogenen Ansatzes erkennen ${ }^{224}$, dessen andere und ebenso pragmatische Basis die steigenden Bedürfnisse des Fernhandels bzw. des Reisens waren:

Obgleich hier von mitteleuropäischen Sprachen gehandelt wird, muß in diesem Konnex an erster Stelle doch der schon 1880 von Géza Kuun edierte Codex Cumanicus der Marciana in Venedig (Cod. Marc. lat. DXLIX) angeführt werden, den man bis vor kurzem sogar mit Francesco Petrarca in Zusammenhang brachte. Es handelt sich um eine lateinisch-persisch-kumanische Enzyklopädie des ausgehenden zwölften Jahrhunderts, deren erster Teil (Fol. 1-110) ein vermutlich von Genuesen verfasstes Dolmetscherbuch (interpreters book) für den italienischen Schwarzmeerhandel ist. Die Ausrichtung weist sich deutlich in einem vielseitigen systematischen Abschnitt, dem eine alphabetische Wortliste vorangeht ${ }^{225}$. Das umfassendste Beispiel merkantiler Ausrichtung in unserem Bereich ist dann das Sprachbuch Georgs von Nürnberg, doch sei hier auch das älteste gedruckte tschechisch-deutsche Lehrbuch von 1527 aus Pilsen genannt, das mir in einer Ausgabe von 1531 zugänglich war. Seine kaufmännisch thematisierten Dialoge weisen inhaltlich entfernte Parallelen mit denen Georgs von 1424 auf ${ }^{226 .}$

Doch zurück zu unseren Quellen: Die Trialogustradition zwischen dem „kaiserlichen“ CVP 2868 und dem Wiener Vokabulardruck von 1513 , ... peregrinantibus apprime vtilis ..., war aufgrund ihrer Geradlinigkeit geeignet, den Bedürfnissen des Handels und der

${ }^{223}$ A. Rossebastiano, Tradition (zit. A. 220) 691.

224 Dazu H. Hänger, Mittelhochdeutsche Glossare und Vokabulare in schweizerischen Bibliotheken bis 1500 (Quellen und Forschungen zur Sprach- und Kulturgeschichte der germanischen Völker N.F. 44). Berlin-New York 1972 bzw. H. Brauner, Die tschechische Lexikographie des 16. Jahrhunderts. Breslau Diss. 1939, 8-18. Dort wird übrigens ein signifikantes Beispiel der Etymologisierung noch in der Art Isidors von Sevilla angeführt: Katherina ist von „Catha = Universum“ und „Ruina“ abgeleitet, weil das teuflische Gebäude durch die Ankunft (der Heiligen) in dieser Welt umstürzte.

${ }^{225}$ Vgl. dazu Peter B. Golden, The Codex Cumanicus, in: Central Asian monuments. Ed. by Hasan B. PAKsoy. Istanbul 1992, besonders 43-53.

226 Vgl. Pausch, Sprachbuch (zit. A. 222) 239-263 und M. BoнатČová, Česko-německá konverzace pro kupce. K počátkům tištěných učebnic $(1531,1540)$. Sbornik Národniho muzea v Praze C/XXI/3 (1976) 120-128. Dazu allgemein J. Fried, Kunst und Kommerz. Über das Zusammenwirken von Wissenschaft und Wirtschaft im Mittelalter, vornehmlich am Beispiel der Kaufleute und Handelsmessen. (Schriften des Historischen Kollegs. Vorträge 32) München 1993, 23-24. 
Herrschenden zu dienen. Die Glossare Mosbachs und Holubařs lassen vermuten, dass zumindest in Mitteleuropa diesseits der Alpen der Bedarf an handfesten volkssprachigen Vokabularen an der soziologischen Spitze gelegentlich sogar früher als im merkantilen Bereich bestanden haben könnte. Das ist ein geistesgeschichtlich nicht uninteressanter Befund. Eine höfisch typisierte Lexikographie läßt sich aber nicht erkennen.

2. Im Lauf dieser Arbeit deutete sich an, daß das Stift Melk im Zusammenhang mit der Erziehung der Habsburger im fünfzehnten Jahrhundert und speziell des Ladislaus Postumus eine besondere Rolle gespielt haben muß, die vielleicht mit der benediktinischen Klosterreform zu tun hat. Hier ist zuerst an den mit dem Ladislausglossar so eng verschränkten Melker Donat zu denken und an den früher erwähnten Tugendtraktat für Maximilian ${ }^{227}$, aber auch an den Umstand, daß die Stiftsbibliothek auffallend viele $L a$ dislaica enthält ${ }^{228}$. Schließlich sei nicht vergessen, daß der berühmte Propst Johann Schlitpacher von Melk aus Ulrich Greimolt, dem informator der älteren Söhne Herzog Albrechts III. von Bayern-(München), den Erziehungstraktat des Aeneas Silvius zukommen ließ ${ }^{229}$.

3. Im Zusammenhang mit der Domus Austriae wird eine Dynamik erkennbar, die sich nicht einfach mit der Herrschaftsfolge von den Luxemburgern an das Haus Habsburg erklären läßt: Noch im fünfzehnten Jahrhundert muß nämlich - trotz aller Langzeitwirkungen der hussitischen Revolution - ein kulturelles Gefälle von Böhmen zu Österreich existiert haben, das sich in Buchausstattungen oder Übernahmen von Schrifttraditionen zeigt. Die minuziösen Forschungen Haidingers haben dazu ein spannendes Kapitel aufgeschlagen, das zu einer neuen Diskussion über die Herkunft der Fraktur führen wird.

Aber auch literarisch besteht eine Abhängigkeit: Das Ladislausvokabular bzw. seine österreichische Kopie wurde vermutlich von Jan Holubař, einem Tschechen, kompiliert, mit dem in Anschluß an den Claretus über einen möglicherweise nur lateinisch-tschechischen Auszug entstandenen Trialogus wirkt eine Prager Initiative Karls IV. bis auf seinen kaiserlichen Nachfolger Maximilian I. weiter. Sie präfiguriert - auch indirekt eine fruchtbare und eng mit Wien verbundene polyglotte Drucktradition im sechzehnten Jahrhundert. Diese Tatsache steht gegen die seinerzeit politisch genehme Betonung einer völligen Abhängigkeit der böhmisch-tschechischen von der deutschen Lexikographie an der Wende zur Neuzeit, was offenbar erst für solche Drucküberlieferungen gilt, die nicht in der Klaretüberlieferung wurzeln ${ }^{230}$.

Im Zusammenhang sei hier nochmals ein eher trauriges wissenschaftsgeschichtliches Kuriosum vermerkt: Obwohl der tschechische Sprachwissenschaftler J. I. Zahradnik das Vokabular für König Ladislaus Postumus bereits 1904 in einer umfassenden Publikation der tschechischen Akademie der Wissenschaften bekannt machte ${ }^{231}$ wird diese bohemi-

${ }_{227}$ Vgl. S. 50.

228 Vgl: etwa A. Lнотsкy, Quellenkunde zur mittelalterlichen Geschichte Österreichs (MIÖG Erg. Bd. XIX) Wien $1963,356-357,372$.

229 S. S. Riezlern, Geschichte Baierns 3. Gotha 1889, 458. Die Handschrift liegt heute als Clm 19651 in der Bayerischen Staatsbibliothek.

230 Vgl. H. Brauner, Die tschechische Lexikographie des 16. Jahrhunderts. Diss. Breslau 1939, 3 und 102-106; zuletzt P. O. Müller, Lexikographie (zit. A. 202) 554

${ }^{231}$ Zahradnik, Slovník (zit. A.7). Ein weiteres Kuriosum ist der Umstand, daß der Prager Gelehrte Václav Hanka auch im Umfeld der böhmischen Lexikographie als Falsifikator tätig war, s. P. Brodský, Neznámé Hankovo falzum v knihovně Národního muzea. Sbornik Národního muzea v Praze CXXX, 1 (1986) $21-28$. 
stische Primärquelle, welche auch völlig neue Einsichten in die Lehrbücher Maximilians, also auf einen Zentralpunkt aristokratischer Bildung und historischen Interesses ermöglicht, unserer Forschung erst jetzt zugänglich. Es ist schwer begreiflich und nur als Folge national(istisch) induzierter Separation ${ }^{232}$ erklärbar, daß seinerzeit - zumal in einer Stadt mit deutscher und tschechischer Karlsuniversität - dieses für die gemeinsame Wissenschaftsgeschichte so bedeutsame Zeugnis von der deutschsprachigen Forschung nicht angenommen wurde. Damit zeigt sich aber auch exemplarisch, daß bohemistische Perspektiven - so oder so - auf Dauer nicht ohne Schaden einsprachig verengt werden dürfen.

4. Die für uns erkennbaren Höhepunkte der Trialogusüberlieferung, mit der Handschrift von 1489 und dem Druck von 1513 lassen - wie bereits angedeutet - den Schluß zu, daß Wien um die Wende zur Neuzeit zu einem Knotenpunkt vulgärsprachlicher Vermittlung geworden war, wozu hier einige Anhaltspunkte gegeben werden sollen. Bei Georg von Nürnberg tritt 1424 noch ein $c h u(n)$ rad von Wien auf, der in Venedig welhisch lernen will ${ }^{233}$. Aber schon 1482 druckt Stephan Koblinger - seine typographische Urheberschaft ist umstritten - in der Donaustadt mit einem italienisch-deutschen Solenissimo Uochabuolista den bahnbrechenden Introito Adams von Rottweil erstmals diesseits der Alpen nach ${ }^{234}$. Dazwischen liegt möglicherweise ein ... frammento di guida turistica italo-tedescho con frasi e paroli di uso corrente ..." der Franziskanerbibliothek zu Aquila, das mit Giovanni da Capestranos Reise nach Wien in Verbindung gebracht wird ${ }^{235}$.

Historischer Hintergrund des Wiener Interesses an lebenden Fremdsprachen ist in erster Linie die periphere Lage im deutschen Sprachraum, noch dazu mit ganz verschiedenzungigen Nachbarn, was sich schon im Alltag, etwa beim Detailhandel, auswirken mußte. Die Vorteile dieser vorgeschobenen Situation lassen sich etwa an der Strahlkraft der Wiener Dombauhütte ablesen, ein Nachteil war der üble Ruf, in dem eine ständig fluktuierende, ihrer Herkunft nach buntscheckige Bevölkerung schon im Mittelalter stand ${ }^{236}$. Anno 1488 hatte man hier einen tschechisch und ungarisch sprechenden Mistrichter aufnehmen müssen, und die Handwerksordnung der Fischer von 1517 belegt, daß auf dem

${ }^{232}$ Zu diesem Begriff und zum Faktum „interethnischer Entwöhnung“ vgl. H. GöBL, Die Sprachensituation in der Donaumonarchie, in: Sprachen in Europa. Sprachsituation und Sprachpolitik in europäischen Ländern. Hrsg. von Ingeborg Ohnheiser, M. Kienpointner und H. Kalb (Innsbrucker Beiträge zur Kulturwissenschaft 30) Innsbruck 1999, besonders 45 und 48.

${ }^{233}$ O. Pausch, Sprachbuch (zit. A. 222) 262. Ebenda, 51 ist angedeutet, daß diese Textstelle wohl das einzige Indiz dafür sein könnte, daß Georg nicht ausschließlich für italienische Schüler schrieb. Man wird in künftigen Diskussionen berücksichtigen müssen, daß es in der kosmopolitischen Serenissima ein Georg nahestehendes (deutsch-italienisches) Skriptorium gegeben haben dürfte, vgl. O. Pausch, Laurin in Venedig, in: Deutsche Heldenepik in Tirol. König Laurin und Dietrich von Bern in der Dietrichepik des Mittelalters (=Beiträge der Neustifter Tagung des Südtiroler Kulturinstituts 1977) Bozen 1979, besonders 202-203.

234 Vgl. dazu A. Rossebastiano, Antichi vocabolari plurilingui d'uso popolare. De Gulden Passer 55 (1977) 106-107.

235 Ebenda, S. 91-92, A.29. Dazu A. Chiappini, Reliquie letterarie Capestranesi. Storia - codici - carte - documenti (Bulletino della R. deputazione Abruzzese di storia patria Ser. III) Aquila 1927, 236, Nr. 369: Repertorio in foglio di frasi usuali latino-tedesche, in due colonne, probabilmente scritte e usufruite dal santo Capistranese nel suo ingresso in Austria-Ungheria (1451).

${ }^{236}$ Zur Bauhütte von St. Stephan vgl. zuletzt 850 Jahre St. Stephan. Symbol und Mitte in Wien 1147-1997 (Historisches Museum der Stadt Wien, Sonderausstellung 226) Wien 1997, besonders 480-481. Zur Reputation der Wiener s. A. Lhotsky, Zeitalter (zit. A. 17) 84. 
Fischmarkt Wiener Bürger als Dolmetscher Beschäftigung fanden. Dreißig Jahre später beschreibt Wolfgang Schmeltzl in seinem Lobspruch der...Statt Wienn in Osterreich... babylonische Verhältnisse im Markttreiben der Stadt ${ }^{237}$.

Ähnlich gemischt dürften die nationes der Alma Mater Rudolphina gewesen sein. Jedenfalls zeigt bereits die Vorrede Thomas Ebendorfers zu seiner Cronica Austrie von 1453 Sorge wegen eines Indifferentismus gegen Österreich in intellektuellen Kreisen, der vermutlich mit der großen Zahl von Scholaren aus Süddeutschland, Böhmen und Ungarn zu tun hatte, von der auch Hartmann Schedels Beschreibung Wiens in seiner Weltchronik berichtet ${ }^{238}$. Und offensichtlich ließ sich auf akademischem Boden gut mit Fremdenfeindlichkeit argumentieren: Am Schluß einer epigrammatischen Invektive bezeichnet Conrad Celtis um 1497 Bernhard Perger, den aus dem damals noch ,,windischen“ Stainz in der Weststeiermark gebürtigen Grammatiker und Superintendenten der Universität, bündig als Non te Germana iam dicam stirpe creatum / Dum spernis patriam, perfide Slave, meam $^{239}$.

Endlich muß die wachsende Rolle Wiens als Residenzstadt und Kristallisationspunkt des habsburgischen Machtbereichs berücksichtigt werden. Hier läßt sich heute die böhmische Trialogustradition am besten belegen, und es ist nur folgerichtig, daß sich auch die politischen Veränderungen nach Mohacs lexikographisch auswirkten, nämlich mit der im vorigen Kapitel genannten sechssprachigen Nomenclatura von 1538 aus der Wiener Offizin Singrieners. Ihr Bearbeiter ist Gabriel Pannonius Pest(h)i, einer der ersten Autoren ungarischsprachiger Druckbücher überhaupt und anerkannter Gelehrter ${ }^{240}$. In der Widmung an den Bischof Franciscus de Wijlak (Ujláki) von Raab - die Auflage Stainhofers von 1568 ist dann dem Grafen Franz von Nádasdy dediziert - bezeichnet Pesti seine Liebe zum Vaterland als Grund für die Arbeit - ein durchaus humanistisches Motiv also - und danach erst den Zweck: Hilfestellung für Personen mit geringen Sprachkenntnissen. Bemerkenswert ist übrigens, daß fast genau hundert Jahre nach Pesti in Wien ein - diesmal spanisch-deutscher - Sprachführer in einer vermutlich spanischen Offizin herauskam, was wohl ebenfalls mit den politisch-dynastischen Verflechtungen der Habsburger zusammenhängt ${ }^{241}$.

${ }^{237}$ Über das Verhältnis der Wiener zur „Außenwelt“ zuletzt F. OpLL, Leben im mittelalterlichen Wien. Wien-Köln-Weimar 1998, S. 64-67.

238 S. Lhotsky, Artistenfakultät (zit. A. 20) 96-97. Zu den akademischen Nationen vgl. P. Uiblein, Die Universität Wien im 14. Und 15. Jahrhundert, in: Das alte Universitätsviertel in Wien, 1385-1985 (= Schriftenreihe des Universitätsarchivs 2) Wien 1985, S.27; E. Spekner, Das geistige Leben in Pest und Buda um die Wende vom 15. zum 16. Jahrhundert, in: G. BiegeL, Budapest im Mittelalter (Schriften des Braunschweigischen Landesmuseums 62) Braunschweig 1991, besonders 317-318; Hartmann Schedel, Liber chronicarum. Das Buch der Croniken und Geschichten. Nürnberg 1493.

239 S. H. Grössing, Die Wiener Universität im Zeitalter des Humanismus von der Mitte des 15. Bis zur Mitte des 16. Jahrhunderts, in: Das alte Universitätsviertel in Wien, 1385-1985. (= Schriftenreihe des Universitätsarchivs 2) Wien 1985, 42-43.

240 Gabriel Pannonius Pesti übersetzte vor der Nomenclatura das Neue Testament und den Äsop ins Madjarische. Vgl. György KóKay, Geschichte des Buchhandels in Ungarn (Geschichte des Buchhandels III) Wiesbaden 1990, 38; József Szinnyei, Magyar írók élete és munkái 10 .Budapest 1905 (Nachdruck 1980/81) 825-827; Res litteraria Hungariae vetus operum impressorum 1473-1600. Budapest 1971, $82-83$ und $87-88$.

${ }^{241}$ Juan Angel de Sumarán, Gramatica y pronunciación alemana y espaňola, espaňiola y alemana, Teutsche vnnd Spannische, Spannische vnd Teutsche Grammatica vnd außsprach. Vienna de Austria: Miguel Riccio Impressor 1634. 
Schon für Thomas Ebendorfer war die Stadt totius domus Austriae capitalis und obwohl die Wiener Vokabulardrucke des 16. Jahrhunderts nur einen Bruchteil der Produktion einschlägiger Druckzentren ausmachen, läßt sich annehmen, daß ab dem späten Mittelalter - neben Predigten, der Legendenliteratur, den ludi usw. - auch vulgärsprachliche Glossare zum wichtigen Schrifttum für alle Gesellschaftsschichten gezählt werden können $^{242}$. Darüberhinaus ist die Nomenclatura sex linguarum - als Gegenstück zur ebenfalls in der Donaustadt gedruckten Trialogusüberlieferung - druckgeschichtlich ein heimischer Traditionszweig, allerdings mit venezianischem Stammbaum: Er hat bis 1568 mindestens fünf oder sechs Auflagen, ausschließlich bei den Wiener Typographen Johann Singriener (Vater und Sohn), Caspar Steinhofer und Philipp Püdinger erlebt ${ }^{243}$.

Die folgenden Bemerkungen zum Gesamtcorpus schließen zunächst an die Einleitung an: Mit den Editionen soll eine Grundlage für weitere und einläßlichere Untersuchungen gelegt werden. Die sprachlichen, quellenkundlichen und historisch-hilfswissenschaftlichen Anmerkungen in dieser Arbeit dienen nur dem Zweck, das Material fürs erste und möglichst offen einzuordnen. Dadurch entgeht man dem Risiko, sich vorschnell und mit unzureichenden Mitteln festzulegen. Es wurde ja bereits auf der Grundlage gewagter biographischer Kombinationen über den Krakauer Drucker Hieronymus Viëtor versucht, den Dictionarius sprachlich der Slowakei zuzuschlagen ${ }^{24}$. Zudem ist jederzeit mit der Möglichkeit zu rechnen, daß neue und präzisierende Materialien zum Thema auftauchen können, was ja die Arbeit von Bedřiška Wižd'álková exemplarisch zeigt. Mein nicht zuletzt auch aus Gründen der Arbeitsökonomie gewählte Prinzip hat sich schon früher, bei der Ausgabe des Sprachbuchs Georgs von Nürnberg bewährt, zu der in der Folge eine ganze Reihe von eingrenzenden - und berichtigenden - Spezialuntersuchungen erschienen ist ${ }^{245}$.

Solches ist auch für die jetzt edierten Vokabulare zu erhoffen, wobei - ganz abgesehen von einer Fülle kulturgeschichtlicher und interlinguistischer Möglichkeiten - die je paarweise Zusammenfassung zweier Traditionen zu je drei Sprachen ihren methodischen Reiz haben müßte. Am Ladislausvokabular mit seiner Kopie im CVP 2945 läßt sich der Prozeß, wie eine „Mischhandschrift“, v.a. im Aufeinanderprall mitteldeutscher mit bairisch-österreichischen Sprachformen, entsteht, hervorragend und bis in semantische Feinheiten beobachten. Der Trialogus wieder mit seiner fast eine Generation jüngeren parallelen Druckfassung und den Varianten aus dem Klaret gibt prächtige Einblicke in den Wandel zweier mitteleuropäischer

${ }^{242}$ Vgl.dazu H. Rupprich, Das Wiener Schrifttum des ausgehenden Mittelalters (SB Österr. Akad. Wiss., Phil.-hist. Kl.228/5) Wien 1954, 17. P. O. Müller, Lexikographie (zit. A. 202) 549, verzeichnet für Strassburg 102, für Leipzig 76, für Augsburg 64, für Wien 8 Editionen

243 Vgl. Brauner, Lexikographie (zit. A. 230) 32-39; Rossebastiano, Antichi vocabolari (zit. A. 213) 247259; P. O. MülLER, Lexikographie (zit. A. 202) 293-

244 Matilda Hayeková, Dejiny slovenských slovníkov do roku 1945. Preßburg 1979, 236; dazu Kę̨ELsKA, Slowniki (zit. A. 2) 22-23.

245 Hier sind u. a. zu nennen: A. Rossebastiano, I „Dialoghi“ di Giorgio da Nurimbergo. Savigliano 1984; A. Karnein, Deutsch als Fremdsprache im 15. Jahrhundert: Das Sprachbuch Meister Jörgs. Jahrbuch Deutsch als Fremdsprache 2 (1976) 1-13; G. Holtus und W. SchweIKard, Elemente gesprochener Sprache in in einem venezianischen Text von 1424: Das italienisch-deutsche Sprachbuch des Georg von Nürnberg, in: Gesprochenes Italienisch in Geschichte und Gegenwart. Hrsg. von G. HoLtus und E. RadTKe (= Tübinger Beiträge zur Linguistik 252). Tübingen 1985, 354-376; H. J. Simon, Zur Problematik einer Geschichte der deutschen Abtönungspartikeln. Fallstudien anhand eines Sprachlehrbuchs von 1424. Sprachwissenschaft 21 (1996) 262-300. 
Vulgärsprachen aber auch der Latinität, vom Ende des Mittelalters zum Beginn der Neuzeit. Es wird - wie schon angedeutet - noch genauer zu bestimmen sein, inwieweit es sich beim Druck um ...eine vermehrte und verbesserte Auflage... ${ }^{246}$ handelt, wo modernisiert und wie die Texte dem sechzehnten Jahrhundert angeglichen sind, das ja - als wichtigste Wegstrecke zum Neuhochdeutschen - auch den Übergang vom Alt- zum Mitteltschechischen markiert ${ }^{247}$. Eine solche Untersuchung hat für den tschechischen Teil bereits B. Wižd'álková angeregt ${ }^{248}$. Wenn ihre Forschungen im typographischen Umfeld des Trialogus einbezogen und erweitert werden können, wird sich wohl ein modellhafter Überblick über die sozial-religiös und durch die neue Reproduktionstechnik bedingte Dynamik sprachlicher Normierungstendenzen entwickeln lassen ${ }^{249}$. Dazu wird sich schließlich die Möglichkeit bieten, das Verhältnis zwischen einem handschriftlichen und gedruckten Vokabulartyp umfassend zu analysieren ${ }^{250}$. Eindeutig jedenfalls widerlegt der Strang vom Claretus zu den Vocabula pro iuvenibus bzw. über den Trialogus bis zum Dictionarius von 1513 und dessen Nachfolgern die Meinung, dass Drucke nur mehr Ausläufer von im wesentlichen handschriftlichen lexikalischen Überlieferungen $\operatorname{seien}^{251}$.

Angesichts unserer polyglotten Traditionen soll ferner die Möglichkeit semantischer wie graphisch-graphematischer Interferenzen zwischen den deutschen und tschechischen Texten zumindest angesprochen werden. Dies empfiehlt sich umsomehr, als eine entsprechende Diskussion, die nie ganz abgebrochen war ${ }^{252}$, von slavistischer Seite gerade wieder in Gang zu kommen scheint ${ }^{253}$. Außerdem liegt bereits interessantes Vergleichsmaterial vor, etwa die Aufzeichnungen Johannes Butzbachs ${ }^{254}$ oder Ausspracheregeln für beide Sprachen in den Traditionen des anonymen Nauczenie kratke obogi řcij Niemecke a Cžeske von $1531^{255}$ bzw. des

246 Wolkan, Geschichte der deutschen Literatur (zit. A. 91) 92.

247 FlajšHans, Klaret (zit. A. 75) XXXII.

248 WiŽந̌Álková, Vokabulař (zit. A. 165) 14.

249 Vgl. dazu Hildegard BoKová, Beobachtungen zur Prager Druckersprache des 16. Jahrhunderts, in: Fata Libellorum. Festschrift für Franzjosef Pensel (= Göppinger Arbeiten zur Germanistik 648) Göppingen 1999, 2; allgemein zuletzt M. Muzikant, Sprachatlanten und die deutsch-tschechischen Sprachkontakte auf lexikalischer Ebene. Zeitschrift für Dialektologie und Linguistik 69/2 (2002), besonders 187.

250 H. Hänger, Mittelhochdeutsche Glossare und Vokabulare in schweizerischen Bibliotheken bis 1500 (Quellen und Forschungen zur Sprach- und Kulturgeschichte der germanischen Völker N. F.44) Berlin-New York 1972, 16-17. Eine konzise Zusammenfassung über die ... Macht des Buchdrucks ... bei Ph. WolfF, Sprachen, die wir sprechen. Ihre Entwicklung aus dem Lateinischen und Germanischen von 100-1500 n. Ch. München 1971, 226-230.

251 Vgl. K. GrubuÚlter, Vocabularius Ex quo. Untersuchungen zu lateinisch-deutschen Vokabularen des Spätmittelalters (Münchener Texte und Untersuchungen zur deutschen Literatur des Mittelalters 17). München 1967,5 .

252 S. E. Skála, Tschechisch-deutsche Sprachkontakte. Acta Universitatis Carolinae. Philologica $2=$ Germanistica Pragensia XII (1994) 14-15.

253 Vgl. T. Berger, Der alttschechische „Umlaut“ - ein slavisch-deutsches Kontaktphänomen ? in: Festschrift für Klaus Trost zum 65. Geburtstag. München 1999, 19-27.

254 Vgl. dazu M. Kомáreк, Alttschechische Gebete im Itinerarium Johannes Butzbachs, in: Deutsch-tschechische Beziehungen im Bereich der Sprache und Kultur. Aufsätze und Studien. Hrsg. Von B. Havránek und R. Fischer (= Abh. Sächs. Akad. Wiss., philol.-hist. Kl. 57/2). Leipzig 1965) 73-86.

255 S. Mirjam Вонатс̌ová, Česko-nĕmecká konverzace pro kupce. K počátkům tištěných učebnic (1531, 1540). Sbornik Národniho muzea v Praze C/XXI/3 (1976) 129-130. Vgl. auch WOLKAN, Geschichte der deutschen Literatur (zit. A. 51) 88; Z. Opava, Bestseller in der frühen Neuzeit. Ausgaben des Gesprächsbüchleins von Ondřej Klatovský (1540), in: Die Volkssprachen als Lerngegenstand im Mittelalter und in der frühen Neuzeit. Akten des Bamberger Symposiums am 18. und 19. Mai 2001. Hrsg. von H. GLücK (Die Geschichte des Deutschen als Fremdsprache 3) Berlin - New York 2002, 60. 
Andreas Klatovský von Dalmanhorst aus Klattau ${ }^{256}$. Die deutschen Texte haben mit Hans Mosers Analyse der maximilianischen Kanzleisprache ein umfassendes tertium comparatio$n i s^{257}$.

Tschechischen Einflüssen mögen immerhin manche ungewöhnliche Schreibungen des Holubar̆glossars entsprechen, wie Enczuldung $\left(32^{\mathrm{v}}, \mathrm{Z} .16\right)^{258}$, Wirlizen $\left(35^{\mathrm{v}}\right.$, Z. 3, zu mhd. verliesen), werzamen $\left(39^{\mathrm{v}}, \mathrm{Z} .5, \mathrm{~B}\right.$ schreibt v(er)samme $\left.(n)\right)$; später korrigiertes werwerfen, werpergen $\left(4^{\mathrm{v}}, \mathrm{Z} .19 / 20\right)$, moltferf $\left(58^{\mathrm{v}}, \mathrm{Z} .4\right)^{259}$, Petrsilie $\left(60^{\mathrm{v}}, \mathrm{Z} .7\right)$, weise statt Triticum/weize $\left(60^{\mathrm{v}}\right.$, Z. 12), vgl. auch deutsch Minsser für tschechisch Minczerz $\left(32^{\text {r }}\right.$, Z. 9, nur CVP 2945) ${ }^{260}$; für den Trialogus sind hier allenfalls Minium/Mynie (3v , Z. 17, im Druck menge) oder - als krasses Beispiel hyperkritischer Rundung - Latro/Vörbörg $\left(42^{v}, \mathrm{Z} .11\right)^{261} \mathrm{zu}$ nennen. Eher umgekehrt beeinflußt könnten etwa Camen für lateinisch Raxum im Holubar̆glossar A und B $\left(41^{\mathrm{r}}\right.$, Z. 6) oder Canonicus/Canobnyk im Trialogus (5 ${ }^{\mathrm{r}}$, Z. 6, Druck kanownik) sein, weil der tschechische Schreibusus - im Unterschied zum (Mittel)deutschen oder Lateinischen - c für Dentalaffrikata einsetzt ${ }^{262}$. Welche Unsicherheiten aber einzukalkulieren sind, zeigt die Schreibform clincet „klingt“ für Resonet (A 39 ${ }^{\mathrm{r}}, \mathrm{Z}$. 19). Bairische b-w-Vertauschungen finden sich in allen deutschen Texten, vgl. im Ladislausstrang $32^{\mathrm{v}}$, Z. 1 B entberffen A Entwerfen, 45 ${ }^{\mathrm{r}}, \mathrm{Z} .18$ B de(r)burgen A Dirwirgen usw., in der Trialogusüberlieferung Beichbischolff (5 $\left.{ }^{\mathrm{r}}, \mathrm{Z} .8\right)$, Consolida-Weybel (!) (13v, Z. 24, im Druck beynwel), Fleischburm (m) (36r, Z. 10) usw. Dazu nun gibt es im tschechischen Text Parallelen, vgl. in der Überlieferung nach Holubař 7v, Z. 2 A

${ }^{256}$ S. Wolkan, Geschichte der deutschen Literatur (zit. A. 91) 88-91. Zuletzt Alena ŠmečKová, Zur individuellen tschechisch-deutschen Zweisprachigkeit in Böhmen. Eine Fallstudie des 16. Jahrhunderts. Acta Universitatis Carolinae. Philologica 2 und $5=$ Germanistica Pragensia XII/XIII (1994 und 1996) 109-117 bzw. 93-103; dieselbe Zum Dialog im deutsch-tschechischen Gesprächsbuch von Ondřej Klatovský, in: Die Volkssprachen als Lerngegenstand im Mittelalter und in der frühen Neuzeit. Akten des Bamberger Symposiums am 18. und 19. Mai 2001. Hrsg. von H. GLÜCK (Die Geschichte des Deutschen als Fremdsprache 3) Berlin - New York 2002, 67-76. Der älteste - und sicher vorbildliche - Versuch, durch kontrastiven Graphemvergleich Ausspracheregeln zweier Zielsprachen zu skizzieren findet sich in Adam von Rottweils Introito e Porta, Venedig 1477. Dazu O. Pausch, Italienisch-deutsche Ausspracheregeln aus dem Quattrocento, in: Ir sult sprechen willekomen. Grenzenlose Mediävistik. Festschrift für Helmut Birkhan zum 60. Geburtstag. Hrsg. von Christa Tuczay, Ulrike Hirhager u. Karin LichtBLAU. Bern usw. 1998, 132-138.

${ }^{257}$ H. Moser, Die Kanzlei Kaiser Maximilians I. Graphematik eines Schreibusus (Innsbrucker Beiträge zur Kulturwissenschaft 5/1-2). Innsbruck 1977.

258 Übernahme des cz-Graphems, neutschechisch č. In vergleichbaren deutschen Texten steht cz nur für die Dentalaffrikata, vgl. Moser, Kanzlei Maximilians (zit. A. 257) 125-126.

259 Vgl. dazu die tschechische Form stokwiss für „Stockfisch“ im Druck von 1513 (32 , Z. 6). Zur Substitution von mhd. v s. A. MaYer, Die deutschen Lehnwörter im Tschechischen (Forschungen zur sudetendeutschen Heimatkunde 3) Reichenberg 1927, 38-39.

${ }^{260}$ Die Schreibung könnte von einem Mißverständnis der Ambivalenz s - z im Deutschen herrühren, vgl. in den Ausspracheregeln des Nauczenie kratke von 1531: S sa z druhdy čtou jako: Seyden čtou voni zeyden. Nach Boнатс̌ová, Česko-nĕmecká konverzace (zit. A. 255) 129.

${ }^{261}$ Vgl. ebenda: Než takové ö, to se vypovida jako u náš: Čechové, neb pás Krönen/Tödten a čtou krenen/tedten. Vermutlich also hyperkritische Schreibung, wie Meize „Mäuse“im Holubar̆glossar (58v3). Hier könnten natürlich auch die (bairischen) Unsicherheiten der Entrundung hineinspielen, vgl. dazu zuletzt $\mathrm{H}$. Boková, Beobachtungen zur Prager Druckersprache des 16. Jahrhunderts, in: Fata Libellorum. Festschrift für Franzjosef Pensel (= Göppinger Arbeiten zur Germanistik 648) Göppingen 1999, 16.

${ }_{262}$ Vgl. Mirjam Bонатс̌ová; Česko-nĕmecká konverzace (zit. A. 255) 130: aber die Behemen brauchen c für $k$ als Latiner und die Deutschen exemplum capitolum vnnd capitel die Behemen schreiben kapitola. 
Vcho B Bcho, 9v, Z. 10 A weczerce B beczerce usw., für den Trialogus seien hier polebka $\left(21^{\mathrm{r}}\right.$, Z. 8), brzed $\left(19^{\mathrm{r}}, \mathrm{Z} .24\right)$ oder swowoda $\left(7^{\mathrm{v}}, \mathrm{Z} .5\right)$ genannt $^{263}$.

Eher überraschend - besonders in Holubař A - ist der gelegentliche Ausfall des initialen h im Deutschen, weil dem Tschechischen die entsprechende Hauchlautartikulation ja geläufig ist ${ }^{264}$, vgl. enteupten (Holubař A 12v , Z. 12, B hat enhauppen), abgesprochn(n) (Holubař B 14 ${ }^{\mathrm{r}}$, Z. 2, A hat richtig Hab gesprochen), Amster (Trialogus 35 $5^{\mathrm{v}}$, Z. 2, im Druck von 1513 hambster), Lasin (Holubař A 9 ${ }^{\mathrm{v}}$, Z. 13, B lashin) aber auch lateinisch aderere statt adherere (Holubařglossar A und B 6r, Z. 6), Ebdomada (Trialogus 1 1 , Z. 29, Druck Hebdomata). Bemerkenswert sind auch die Hyperkritismen hoberst (Holubař A 45 , Z. 1, B obrist) bzw. he (A und B 29r, Z.7). Die Form chuoden für "Hoden" (Ladislausglossar 65" , Z. 14) wurde bereits auf S. 33 erörtert Abschließend sei die Häufung von Metathesen v.a. in der Ladislaustradition B hervorgehoben : 16 ${ }^{\mathrm{r}}$, Z. 2 A Enphlien B enphilen, 28 ${ }^{\mathrm{r}}$, Z. 5 A Heuschreck B Heuscherck, 31 ${ }^{\mathrm{v}}$, Z. 6 A Schalk B Schlack, 39", Z. 17 A Schtrofen B Starffn(n), 41'v, Z. 3 A Schilt B Schlitt (!), vgl. dazu 4r, Z. 12 A Przitelnicze B przitelince, 8, Z. 4 A Steblo B stelbo, 22 $2^{\mathrm{r}}$ Z. 10 A Jadro zrno B Jardo zrno, 25 ${ }^{\mathrm{r}}$, Z. 14 A wnitrz B wintrz u.a. Sekundär tschechisch beeinflußt scheint deutsch pratt, tschechisch Bradadicze zu lateinisch dolabrum (15, Z. 3 A schreibt Bart, vgl. mhd. barte).

Noch eine Beobachtung muß hier wiederholt werden, weil sie für die künftige Beurteilung einschlägiger Quellen einkalkuliert werden sollte. Es ist gewiß erstaunlich, wie sorglos - bei gleichzeitigem Halten eines ansprechenden Formniveaus - von den Kopisten bzw. auch Drukkern mit dem jeweiligen Glossartext verfahren wurde, so zwar, daß in vielen Fällen die Fehler über alle Sprachen verteilt sind. Das war in der Lexikographie vor und nach 1500 offensichtlich nicht ungewöhnlich. Als Pariser Beispiel seien die Inkunabeln des Catholicum abbreviatum von Antoine Caillaut erwähnt, und noch das 1532 von Johann Peck gedruckte Pilsner Vokabular, ein Werk in der Trialogustradition, muß nicht nur bei Slawisten Kopfschütteln auslösen $^{265}$. Aber sogar der Cod. Pal. lat. 1789 des ausgesprochenen Spezialisten Johann von Mosbach-Neumarkt, in dem sich - wie im CVP 2945 - ein Korrektor nachweisen läßt, ist vor Irrtümern nicht ganz gefeit ${ }^{266}$.

Man sollte dabei - neben dem Fehlen einer Devotio ad litteram in den Schreibstuben - allgemein in Rechnung stellen, wiesehr sich im fünfzehnten Jahrhundert vulgärsprachige Polyglottie flexibel auf die parole und auf das ,Nebeneinander zweier Sprachen in einer Person“"konzentriert haben muß ${ }^{267}$, ein Faktum, das man sich nach einer Periode ,,interethnischer Entwöhnung" schwer vorstellen kann. Man wird am Beispiel unserer regentenbezogenen Texte aber auch Zusammenhänge mit der inferioren sozialen Stellung von Kanzleischreibern nicht ausschließen können. Über die tristen Verhältnisse am Wiener Neustädter

263 Auf diese b-w-Vertauschungen im tschechischen Text hat übrigens schon MeNčík, Prešpurský slovník (zit. A.162) XXVII hingewiesen.

264 Vgl. dazu A. MaYer, Die deutschen Lehnwörter im Tschechischen (Forschungen zur sudetendeutschen Heimatkunde 3) Reichenberg 1927, 43-44.

${ }^{265} \mathrm{Vgl}$. dazu J. Sнaw, The printed dictionaries in France before 1539. Toronto (Electronic Book) 1997, XXX bzw. KęDelska, Slowniki (zit. A. 2) 22.

266 Vgl. Křesślková, Vocabolario Quadrilingue (zit. A. 221) IV-V.

267 A. ŠmečKová, Zur individuellen tschechisch-deutschen Zweisprachigkeit in Böhmen. Eine Fallstudie des 16. Jahrhunderts. Acta Universitatis Carolinae. Philologica 2 = Germanistica Pragensia XII (1994) 109. Vgl. auch Fn. 192. 
Hof Kaiser Friedrichs III. zeichnet Aeneas Silvius Piccolomini ein plastisches Bild ${ }^{268}$, und auch unter Maximilian I. scheint gegolten zu haben, daß die Kanzlisten „... in einem gewissen Sinn tatsächlich nur als "Schreibmaschinen“ benutzt wurden ..." ${ }^{269}$.

Insgesamt mahnt die geringe inhaltliche Sorgfalt lexikalischer Übertragungen, die schon vor eineinhalb Jahrhunderten Editoren resignieren ließ $3^{270}$, auch zu Vorsicht bei „nationaler“ Zuschreibung im heutigen Sinn, immer - wie bereits angedeutet - auch in Hinblick auf die Flexibilität bilingualer Kompetenzen in einer noch wenig alphabetisierten Zeit ${ }^{271}$. Die Unzuverlässigkeit kopialer Überlieferung hat niemand geringeren als I. V. Jagić in Zusammenhang mit Menčíks Ausgabe des Preßburger Klaretglossars zu dem Satz veranlaßt „... ich muß offen gestehen, daß ich selten eine Seite des gedruckten Textes las, ohne auf Rätsel zu stossen, deren

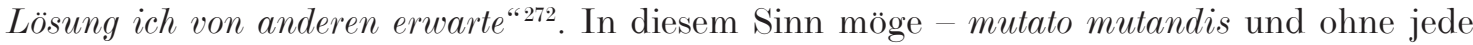
Ironie - auch die vorliegende Arbeit aufgenommen werden.

268 S. G. Voigt, Enea Silvio Piccolomini als Papst Pius II. und sein Zeitalter 1.Berlin 1856, 275.

269 H. Moser, Die Kanzlei Kaiser Maximilians I. Graphematik eines Schreibusus (Innsbrucker Beiträge zur Kulturwissenschaft 5/1). Innsbruck 1977. 29.

270 K. J. SchröER, Lateinisch-deutsches Vocabular des XV. Jahrhunderts (Jahresprogramm der öffentlichen Ober-Realschule der $k$. Freistadt Preßburg 9). Preßburg 1859, 62.

271 Bellmann, Slavoteutonica (zit. A. 85) 9-61. Vgl. dazu auch die aus dialektologischer Praxis gewonnene Systematisierung bei E. Kranzmayer, Die bairischen Kennwörter und ihre Geschichte. Wien 1966 , $24-28$

272 J. V. JAGić, Rezension von F. Menčíks Vocabularium latinobohemicum Posoniensis. Archiv für slavische Philologie 14 (1893) 310. 